\title{
INVERSES AND PARAMETRICES FOR RIGHT-INVARIANT PSEUDODIFFERENTIAL OPERATORS ON TWO-STEP NILPOTENT LIE GROUPS
}

BY

KENNETH G. MILLER

\begin{abstract}
Let $P$ be a right-invariant pseudodifferential operator with principal part $P_{0}$ on a simply connected two-step nilpotent Lie group $G$ of type $H$. It will be shown that if $\pi\left(P_{0}\right)$ is injective in $\varsigma_{\pi}$ for every nontrivial irreducible unitary representation $\pi$ of $G$, then $P$ has a pseudodifferential left parametrix. For such groups this generalizes the Rockland-Helffer-Nourrigat criterion for the hypoellipticity of a homogeneous right-invariant partial differential operator on $G$. If, in addition, $\pi(P)$ is injective in $\varsigma_{\pi}$ for every irreducible unitary representation of $G$, it will be shown that $P$ has a pseudodifferential left inverse. The constructions of the inverse and parametrix make use of the Kirillov theory, their symbols being obtained on the orbits individually and then pieced together.
\end{abstract}

1. Introduction. Let $\mathcal{G}$ be a two-step nilpotent Lie algebra: $\mathcal{G}=\mathcal{G}_{1} \oplus \mathcal{G}_{2}$ where $[\mathcal{G}, \mathcal{G}] \subset \mathcal{G}_{2}$ and $\left[\mathcal{S}_{2}, \mathcal{G}\right]=0$. Let $G$ be the corresponding connected, simply connected Lie group. Identifying $\mathcal{G}^{*}$ with $\mathfrak{S}_{1}^{*} \times \mathcal{G}_{2}^{*}$ we will frequently denote an element $\xi \in \mathcal{G}^{*}$ by $\xi=(\eta, \zeta)$ with $\eta \in \mathcal{S}_{1}^{*}, \zeta \in \mathcal{S}_{2}^{*}$. We assume that some norm is given on $\mathcal{S}^{*}$. Define $\Phi$ on $\mathcal{G}^{*}$ by $\Phi(\eta, \zeta)^{2}=|\eta|^{2}+\left(|\zeta|^{2}+1\right)^{1 / 2}$. As in [17], for $x \in \mathcal{G}$ and a function $p$ defined on $\mathcal{S}^{*}$, let

$$
D_{x} p(\xi)=\left.\frac{d}{d t} p\left(\xi+(\operatorname{ad} t x)^{*} \xi\right)\right|_{t=0}
$$

when the derivative exists. Note that $D_{x} p(\xi)$ is a derivative of $p$ in a direction parallel to $\theta_{\xi}$, where $\theta_{\xi}$ denotes the orbit of $\xi$ under the coadjoint action of $G$ on $\mathcal{G}^{*}$. Given $m \in \mathbf{R}$ and $\Omega$ an open subset of $\mathcal{G}^{*}, S^{m}(\Omega)$ is the set of complex valued functions $p$ defined on $\Omega$ such that, for every $k$ and every choice of $x_{1}, \ldots, x_{k}$ in $\mathcal{G}$, $D_{x_{1}} \cdots D_{x_{k}} p$ exists and is continuous on $\Omega$, and

$$
\left|D_{x_{1}} \cdots D_{x_{k}} p(\xi)\right| \leqslant C_{k} \Phi(\xi)^{m-k} \prod_{1}^{k}\left|\operatorname{ad} x_{j}^{*} \xi\right|
$$

for all $\xi \in \Omega$.

$S_{0}^{m}\left(\mathcal{G}^{*}\right)$ is the set of $p \in S^{m}\left(\mathcal{G}^{*}\right)$ such that $p=p_{0}+p_{1}$ where $p_{1} \in S^{m-\varepsilon}\left(\mathcal{G}^{*}-\{0\}\right)$ for some $\varepsilon>0$ and $r^{-m} p_{0}\left(r \eta, r^{2} \zeta\right)=p_{0}(\eta, \zeta)$ for $r>0$. Let $S^{-\infty}(G)$ be the set of complex valued functions $u$ defined on $G$ such that $F^{-1}(u \circ \exp ) \in S^{k}\left(\mathcal{G}^{*}\right)$ for all $k \in \mathbf{R}$. Here $F$ denotes the Fourier transform and exp: $\mathcal{G} \rightarrow G$ is the exponential

Received by the editors November 22, 1982.

1980 Mathematics Subject Classification. Primary 35S05, 35H05; Secondary 58G15. 
map. For $p \in S^{m}\left(\mathcal{G}^{*}\right), \lambda(p): S^{-\infty}(G) \rightarrow S^{-\infty}(G)$ is defined by $\lambda(p) u=$ $(F p \circ \log ) * u$. As shown in [17], given $p \in S^{m}\left(\mathcal{G}^{*}\right)$ and $q \in S^{k}\left(\mathcal{G}^{*}\right)$, there is an element, denoted $p \# q$, of $S^{m+k}\left(\varrho^{*}\right)$ such that $\lambda(p \# q)=\lambda(p) \lambda(q)$.

If $\xi \in \mathcal{S}^{*}, \tilde{V} \subset \mathcal{G}$ is a subalgebra maximally subordinate to $\xi$ and $V$ is a subspace of $\mathcal{G}$ complementary to $\tilde{V}$, let $\pi=\pi_{\xi, V, \tilde{V}}$ be the irreducible unitary representation of $G$ on $L^{2}(V)$ as defined in [8, 16 or 17]. Every irreducible unitary representation of $G$ is unitarily equivalent to at least one such representation. For $x \in \mathcal{G}$ let

$$
\pi(x)=-\left.i \frac{d}{d t} \pi(\exp t x)\right|_{t=0} .
$$

Then $\pi(x)$ is a differential operator on $V$ for which the symbol is a real affine function on $V \times V^{*}$. Define $\psi_{\pi}: V \times V^{*} \rightarrow \mathcal{G}^{*}$ by

$$
\left\langle\psi_{\pi}(t, \tau), x\right\rangle=\operatorname{sym} \pi(x)(t, \tau) .
$$

$\psi_{\pi}$ is a symplectomorphism of $V \times V^{*}$ onto that orbit $\theta_{\pi}$ of the coadjoint action of $G$ on $\mathcal{G}^{*}$ which corresponds to $\pi$ in the Kirillov theory (see [17]). If $p \in S^{m}\left(\mathcal{G}^{*}\right)$, define $\pi(p)$ to be the pseudodifferential operator on $V$ with Weyl symbol $p \circ \psi_{\pi}$. In [17] it is shown that $\pi(p \# q)=\pi(p) \pi(q)$ for $p \in S^{m}\left(\mathcal{G}^{*}\right), q \in S^{k}\left(\mathcal{G}^{*}\right)$ and furthermore $\pi(p)$ agrees with the usual definition of $\pi(P)$ when $P=\lambda(p)$ is a partial differential operator, i.e. when $p$ is a polynomial.

For $\zeta \in \mathcal{G}_{2}^{*}$ define the alternating bilinear form $B_{\zeta}$ on $\mathcal{G}_{1}$ by $B_{\zeta}(x, y)=\langle\zeta,[x, y]\rangle$. Following Lévy-Bruhl [12] we say that $\mathcal{G}$ is of type $H$ if $\operatorname{rank} B_{\zeta}$ is constant for $\zeta \neq 0$. Note that this is less restrictive than the statement of type $H$ or hypothesis $H$ as given in [14 or 19], where $\mathcal{G}$ is said to be of type $H$ if $B_{\zeta}$ is nondegenerate for $\zeta \neq 0$. For example, if $\mathcal{G}$ is the free two-step Lie algebra on three generators (a six-dimensional Lie algebra), then $\operatorname{rank} B_{\zeta}=2$ for $\zeta \neq 0$, while $\operatorname{dim} \mathcal{G}_{1}=3$. The primary obstructions to developing the main results of this paper for general step two groups lie in proving Lemma 8 and Corollary 1 , in general.

In the following theorems the "trivial representation" is the one-dimensional identity representation. $\mathcal{S}_{\pi}$ is the space of $C^{\infty}$ vectors for $\pi: \delta_{\pi}=\delta(V)$ when $\pi=\pi_{\xi, V, \tilde{V}}$

THEOREM 1. Let $\mathcal{G}$ be of type $H$ and let $p \in S_{0}^{m}\left(\mathcal{G}^{*}\right)$. If $\pi(p)$ is injective on $\varsigma_{\pi}$ for every irreducible unitary representation $\pi$ of $G$, and $\pi\left(p_{0}\right)$ is injective on $\varsigma_{\pi}$ for every nontrivial irreducible unitary $\pi$, then there is a $q \in S^{-m}\left(\mathcal{G}^{*}\right)$ such that $\lambda(q) \lambda(p)=I$.

THEOREM 2. Let $\mathcal{G}$ be of type $H$ and let $p \in S_{0}^{m}\left(\mathcal{G}^{*}\right)$. If $\pi\left(p_{0}\right)$ is injective on $\mathfrak{S}_{\pi}$ for every nontrivial irreducible unitary representation $\pi$ of $G$, then there is a $q \in S^{-m}\left(\mathcal{G}^{*}\right)$ such that $q \# p-1 \in S^{-k}\left(\mathcal{G}^{*}\right)$ for all $k$, and hence $\lambda(q) \lambda(p)-I: \mathcal{E}^{*}(G) \rightarrow \mathcal{\varepsilon}(G)$.

Since $\lambda(\bar{p})$ is the formal adjoint of $\lambda(p)$, and $\pi(\bar{p})$ is the formal adjoint of $\pi(p)$ for each $\pi$, the above theorems, when applied to $\bar{p}$, give sufficient conditions for the existence of a right inverse or right parametrix for $\lambda(p)$.

The statement that $q \in S^{m}\left(\mathcal{G}^{*}\right)$ is not strong enough to imply that $\hat{q} \in$ $C^{\infty}(\mathcal{G}-\{0\})$, i.e. that $\lambda(q)$ be pseudolocal. (For example, when $G$ is abelian, $q \in S^{0}\left(\mathcal{G}^{*}\right)$ if and only if $q$ is continuous and bounded.) We introduce the following 
more restricted classes of symbols: $p \in \tilde{S}^{m}\left(\mathcal{G}^{*}\right)$ if $p \in \mathcal{E}\left(\mathcal{G}^{*}\right)$ and there is an $\varepsilon$, $0<\varepsilon \leqslant 2$, such that for all $\alpha$ and $\beta$

$$
\left|D_{\eta}^{\alpha} D_{\zeta}^{\beta} p(\eta, \zeta)\right| \leqslant C_{\alpha \beta} \Phi(\eta, \zeta)^{m-|\alpha|-\epsilon \beta \mid}
$$

for all $(\eta, \zeta) \in \mathcal{G}^{*}$. In connection with Theorem 2 , we prove that if $p \in \tilde{S}_{0}^{m}\left(\mathcal{G}^{*}\right)$, then $q \in \tilde{S}^{-m}\left(\mathcal{G}^{*}\right)$, hence $\lambda(q)$ is pseudolocal and $\lambda(p)$ is hypoelliptic. We also have the following converse.

THEOREM 3. Let $G$ be step two nilpotent. Suppose $p \in \tilde{S}^{m}\left(\mathcal{S}^{*}\right)$ is homogeneous in the sense that for some $c>0, p\left(r \eta, r^{2} \zeta\right)=r^{m} p(\eta, \zeta)$ for all $(\eta, \zeta)$ such that $|\eta|+|\zeta|^{1 / 2}$ $=c$ and all $r \geqslant 1$. If $\lambda(p)$ is hypoelliptic, then $\pi(p)$ is injective on $\S_{\pi}$ for every irreducible unitary representation $\pi$ for which $\min \left\{|\eta|+|\zeta|^{1 / 2}:(\eta, \zeta) \in 0_{\pi}\right\} \geqslant c$.

Note that the homogeneity condition is needed, for there exist polynomials $p \in S_{0}^{m}\left(\mathcal{G}^{*}\right)$ for which $\lambda(p)$ is hypoelliptic but $\pi\left(p_{0}\right)$ is not injective for all nontrivial $\pi$, for example $p\left(\eta_{1}, \eta_{2}, \zeta\right)=\eta_{1}^{2}+i \eta_{2}$ on the Heisenberg group. $(\lambda(p)$ is hypoelliptic by Hörmander [10] or Miller [16].)

Theorems 2 and 3 are, of course, analogs of the following theorem of Helffer and Nourrigat: If $P$ is a homogeneous left invariant partial differential operator on a nilpotent group $G$, then a necessary and sufficient condition for $P$ to be hypoelliptic is that $\pi(P)$ be injective on $\widehat{S}_{\pi}$ for all nontrivial irreducible unitary representations $\pi$ of $G$. This theorem was proved in full generality in [9]. It was first given for the Heisenberg group by Rockland in [18]. Other proofs of various degrees of generality are given in $[4,3,7,15$, and 8$]$. The necessity of the given condition was proved in full generality by Beals [3], and our proof of Theorem 3 is only a modification of his proof. For the Heisenberg group $H^{n}$, in studying local solvability, Geller [6] has carried the analysis further and shown that if $P$ is elliptic in the generating directions (i.e. $\pi(P)$ is invertible for the nonzero one-dimensional representations), then there are homogeneous distributions $E$ and $R$ such that $P E=\delta-R, u \rightarrow u * R$ is the projection in $L^{2}\left(H^{n}\right)$ onto $\left[P S\left(H^{n}\right)\right]^{\perp}$, and $E$ is real analytic away from 0 . Melin [13] recently gave a parametrix version of this result, which incidentally allows for the treatment of some situations when $P$ is not homogeneous. His construction makes use of the Weyl pseudodifferential operator calculus at the orbit level, as does the construction in the present paper.

When $G$ is step two nilpotent the existence of a pseudodifferential left parametrix for all left-invariant homogeneous hypoelliptic partial differential operators was proved in [16]. That proof was based on certain a priori estimates which, by a general theorem of Beals [2], imply the existence of a parametrix. A similar proof could be given for those invariant pseudodifferential operators for which the symbol $p$ is smooth and satisfies estimates of the form $\left|D^{\alpha} p(\xi)\right| \leqslant C_{\alpha} \Phi(\xi)^{m-|\alpha|}$ for partial derivatives in all directions.

The present constructions of an inverse (Theorem 1) or a parametrix (Theorem 2) are more direct. The idea for Theorem 1 is, in fact, quite simple: Each of the operators $\pi(p)$ has an inverse which is a pseudodifferential operator on $V$ (Lemma 7). Let $q_{\pi}$ be its Weyl symbol. Define $q$ on $\theta_{\pi}$ by $\left.q\right|_{\pi}=q_{\pi} \circ \psi_{\pi}^{-1}$. The difficulty is to show that $q \in S^{-m}\left(\mathcal{G}^{*}\right)$. 
To construct a parametrix for $\lambda(p)$ under the hypotheses of Theorem 2 it does not suffice to simply let $\left.q\right|_{\mathfrak{O}_{\pi}}=q_{\pi} \circ \psi_{\pi}^{-1}$ where $q_{\pi}$ is a parametrix for $\pi(p)$. The problem is that the remainder terms obtained by the standard parametrix construction behave badly as the orbits get far away from 0 . Indeed, as we show below, there is a parametrix $q_{\pi}$ for $\pi(p)$ for all infinite-dimensional $\pi$ under the weaker hypothesis that $\pi\left(p_{0}\right)$ be invertible for all nontrivial one-dimensional representations $\pi$, which is not a sufficient condition for the hypoellipticity of $\lambda(p)$. The hypotheses of Theorem 2, however, imply in addition that there is a $C$ such that $\pi(p)$ is invertible for those representations for which $\left|\zeta_{\pi}\right| \geqslant C$. (If $\theta$ is the orbit for $\pi$, then $\zeta_{\pi}=\xi_{\mathcal{G}_{2}}$ is independent of the choice of $\xi \in \mathcal{O}$.) The symbol of the parametrix in Theorem 2 is then obtained by piecing together orbitwise parametrices for those orbits for which $\left|\zeta_{\pi}\right|$ is small together with orbitwise inverses for those orbits for which $\left|\zeta_{\pi}\right|$ is large.

2. Lemmas. We begin by deriving some coercive estimates for the symbol and principal symbol. Let $\langle\zeta\rangle=\left(|\zeta|^{2}+1\right)^{1 / 2}$ and $\|\eta, \zeta\|=|\eta|+|\zeta|^{1 / 2}$.

LEMma 1. If $p \in S_{0}^{m}\left(\mathcal{G}^{*}\right)$ and $\pi\left(p_{0}\right) \neq 0$ for all nontrivial one-dimensional representations $\pi$ of $G$ (i.e. $p_{0}(\eta, 0) \neq 0$ for all $\eta \in \mathcal{G}_{1}^{*}, \eta \neq 0$ ), then there exist $C$ and $c$, $0<c<1$, such that

$$
\begin{gathered}
\left|p_{0}(\eta, \zeta)\right| \geqslant c\|\eta, \zeta\|^{m} \quad \text { if }|\zeta| \leqslant c\|\eta, \zeta\|^{2} \neq 0 ; \\
|p(\eta, \zeta)| \geqslant c\|\eta, \zeta\|^{m} \quad \text { if }|\zeta| \leqslant c\|\eta, \zeta\|^{2} \text { and }\|\eta, \zeta\| \geqslant C ; \\
u\left|p_{0}(\eta, \zeta)\right| \geqslant c \Phi(\eta, \zeta)^{m} \quad \text { if }\langle\zeta\rangle \leqslant c \Phi(\eta, \zeta)^{2} ; \\
|p(\eta, \zeta)| \geqslant c \Phi(\eta, \zeta)^{m} \quad \text { if }\langle\zeta\rangle \leqslant c \Phi(\eta, \zeta)^{2} .
\end{gathered}
$$

Proof. By the continuity of $p_{0}$ there is a $c, 0<c<1$, such that

$$
\left|p_{0}(\eta, \zeta)\right| \geqslant c \text { if }\|\eta, \zeta\|=1 \text { and }|\zeta| \leqslant c
$$

For $(\eta, \zeta) \in \mathcal{G}^{*},(\eta, \zeta) \neq(0,0)$, let $r=\|\eta, \zeta\|^{-1}$. Then (2.1) follows from (2.5) and the homogeneity of $p_{0}$. Since $r^{-m} p\left(r \eta, r^{2} \zeta\right)$ converges to $p_{0}(\eta, \zeta)$ uniformly on the compact set $\{(\eta, \zeta):\|\eta, \zeta\|=1,|\zeta| \leqslant c\}$ as $r \rightarrow \infty$, there is a $C$ such that $r^{-m} p\left(r \eta, r^{2} \zeta\right) \geqslant c$ if $\|\eta, \zeta\|=1,|\zeta| \leqslant c$ and $r \geqslant C$. This implies (2.2). For $c$ and $C$ satisfying (2.2) let $c_{1}=\min \left\{c, C^{-2}\right\} / 2$. Suppose $\langle\zeta\rangle \leqslant c_{1} \Phi(\eta, \zeta)^{2}$. Then $|\eta|^{2} \geqslant$ $\left(c_{1}^{-1}-1\right)\langle\zeta\rangle \geqslant\left(c^{-1}-1\right)|\zeta|$, which implies both $\|\eta, \zeta\|^{2} \geqslant C^{2}$ and $|\zeta| \leqslant c\|\eta, \zeta\|^{2}$. Thus, $|p(\eta, \zeta)| \geqslant c\|\eta, \zeta\|^{m}$ and, moreover, since $\|\eta, \zeta\|$ is bounded below, there is a $C_{1}$ such that $\Phi(\eta, \zeta)^{m} \leqslant C_{1}\|\eta, \zeta\|^{m}$. This proves (2.4). Similarly, (2.1) implies (2.3).

LEMMA 2. If $p$ satisfies the hypotheses of Lemma 1 and, in addition, $\pi(p) \neq 0$ for all one-dimensional representations $\pi$ of $G$, then there is a $c, 0<c<1$, such that

$$
|p(\eta, \zeta)| \geqslant c \Phi(\eta, \zeta)^{m} \quad \text { if }|\zeta| \leqslant c \Phi(\eta, \zeta)^{2} .
$$

Proof. This follows from (2.4) and the fact that given $C$ there exists $c_{1}>0$ such that $|p(\eta, \zeta)| \geqslant c_{1}$ if $|\zeta| \leqslant c_{1}$ and $|\eta| \leqslant C$. 
Let $V$ be a $d$-dimensional real vector space with a linear coordinate system. If $p, q \in C^{\infty}\left(V \times V^{*}\right)$, define

$$
\{p, q\}_{j}(x, \xi)=\left.\left(\sum_{i=1}^{d}\left(\frac{\partial^{2}}{\partial \xi_{i} \partial y_{i}}-\frac{\partial^{2}}{\partial \eta_{i} \partial x_{i}}\right)\right)^{j} p(x, \xi) q(y, \eta)\right|_{(y, \eta)=(x, \xi)}
$$

Let $p \in S^{m}\left(\mathcal{G}^{*}\right), q \in S^{k}\left(\mathfrak{G}^{*}\right)$. For any orbit $\mathcal{G}$ of the coadjoint action of $G$ on $\mathcal{G}^{*}$, define $\{p, q\}_{j}$ on $\theta$ by

$$
\left.\{p, q\}_{j}\right|_{c}=\left\{p \circ \psi_{\pi}, q \circ \psi_{\pi}\right\}_{j} \circ \psi_{\pi}^{-1},
$$

where $\pi=\pi_{\xi, V, \tilde{V}}$ is an irreducible unitary representation corresponding to $\theta$ in the Kirillov theory. The definition is independent of the choice of the particular representation $\pi$, since $\psi_{\pi}$ is a symplectomorphism and (2.7) is a symplectic invariant. Let $\Omega=\mathcal{G}_{1}^{*} \times\left(\mathcal{G}_{2}^{*}-\{0\}\right)$. As shown in [17], it follows from Theorem 4.2 of Hörmander [11] that

$$
|\zeta|^{-j}\{p, q\}_{j} \in S^{m+k-2 j}(\Omega)
$$

and, furthermore, given $J>0$,

$$
p \# q=\sum_{j<J}(2 i)^{-j}\{p, q\}_{j} / j !+r_{J}
$$

where $|\zeta|^{-J} r_{J} \in S^{m+k-2 J}(\Omega)$.

Lemma 3. Let $\Omega=\mathcal{G}_{1}^{*} \times\left(\mathcal{G}_{2}^{*}-\{0\}\right)$. If $p$ satisfies the hypotheses of Lemma 2 , then there is a $b \in S^{-m}(\Omega)$ such that $|\zeta|^{-J}(b \# p-1)$ and $\mid \zeta^{-J}(p \# b-1)$ are in $S^{-2 J}(\Omega)$ for all $J \geqslant 0$.

Proof. The proof is a modification of the standard parametrix construction. Choose $F \in C^{\infty}(\mathbf{R})$ such that $F(r) \equiv 1$ if $|r| \geqslant 2$ and $F(r) \equiv 0$ if $|r| \leqslant 1$. For $|\zeta| \neq 0$, define $\phi_{j}(\eta, \zeta)=F\left(\varepsilon_{j} \Phi(\eta, \zeta)^{2}|\zeta|^{-1}\right)$, where $\left\{\varepsilon_{j}\right\}_{0}^{\infty}$ is a decreasing sequence of positive numbers such that $\varepsilon_{j} \leqslant c$ (the $c$ of Lemma 2) for all $j \geqslant 0$ and $\lim _{j \rightarrow \infty} \varepsilon_{j}=0$. Further restrictions will be placed on $\left\{\varepsilon_{j}\right\}$ in the course of the proof. Let $b_{0}=\phi_{0} p^{-1}$ and define $b_{j}$ recursively by

$$
b_{j}=-\phi_{j} p^{-1} \sum_{k=1}^{j}\left\{b_{j-k}, p\right\}_{k}(2 i)^{-k} / k !
$$

By Lemma $2, b_{0} \in S^{-m}(\Omega)$ and by induction, $|\zeta|^{-j} b_{j} \in S^{-m-2 j}$ for all $j$. Because $\varepsilon_{j} \rightarrow 0$ we can define $b=\sum_{j=0}^{\infty} b_{j} \in S^{-m}(\Omega)$, since the sum is locally finite on $\Omega$. Given $k, J$ and $j>J$ there is a $C_{k j J}$ such that

$$
\left|D_{x_{1}} \cdots D_{x_{k}} b_{j}(\eta, \zeta)\right| \leqslant C_{k j J}|\zeta|^{J+1} \Phi(\eta, \zeta)^{-m-k-2 J-2} \prod_{i=1}^{k}\left|\operatorname{ad} x_{i}^{*}(\eta, \zeta)\right|
$$

for any $x_{1}, \ldots, x_{k} \in \mathcal{G}$. On the support of $b_{j},|\zeta| \Phi(\eta, \zeta)^{-2} \leqslant \varepsilon_{j}$, so by choosing $\varepsilon_{j} \leqslant 2^{-j} \min \left\{C_{k j J}^{-1}: k \leqslant j, J \leqslant j\right\}$ we obtain

$$
|\zeta|^{-J} \sum_{j>J} b_{j} \in S^{-m-2 J}(\Omega)
$$

for all $J$. 
For any $\alpha$ and $j, D_{\eta}^{\alpha}\left(1-\phi_{j}\right)$ is a linear combination of terms of the form

$$
F^{(k)}\left(\varepsilon_{j} \Phi(\eta, \zeta)^{2}|\zeta|^{-1}\right) \prod_{i=1}^{k}\left(|\zeta|^{-1} D^{\alpha(i)} \Phi(\eta, \zeta)^{2}\right),
$$

where $\Sigma \alpha(i)=\alpha$. If $|\alpha|>0,|\zeta|^{-1} \Phi(\eta, \zeta)^{2}$ is bounded above and below on the support of $D^{\alpha}\left(1-\phi_{j}\right)$; thus for any $J$

$$
\left|D_{\eta}^{\alpha}\left(1-\phi_{j}\right)(\eta, \zeta)\right| \leqslant C_{\alpha j J} \Phi(\eta, \zeta)^{-|\alpha|}\left(|\zeta|^{-1} \Phi(\eta, \zeta)^{2}\right)^{-J}
$$

Hence,

$$
|\zeta|^{-J}\left(1-\phi_{j}\right) \in S^{-2 J}(\Omega) \text { for all } j \text { and } J .
$$

For any $j, J \geqslant 1,(2.10)$ and (2.12) imply

$$
\sum_{k=0}^{j}\left\{b_{j-k}, p\right\}_{k}(2 i)^{-k} / k !=\left(1-\phi_{j}\right) \sum_{k=1}^{j}\left\{b_{j-k}, p\right\}_{k}(2 i)^{-k} / k ! \in|\zeta|^{J} S^{-2 J}(\Omega) \text {. }
$$

Hence,

$$
\begin{aligned}
\sum_{j=0}^{J}\left(b_{j} \# p\right) & =\sum_{j=0}^{J} \sum_{k=0}^{j}\left\{b_{j-k}, p\right\}_{k}(2 i)^{-k} / k !+|\zeta|^{J} S^{-2 J}(\Omega) \\
& =\phi_{0}+|\zeta|^{J} S^{-2 J}(\Omega)=1+|\zeta|^{J} S^{-2 J}(\Omega) .
\end{aligned}
$$

It now follows from (2.11) that

$$
|\zeta|^{-J}(b \# p-1) \in S^{-2 J}(\Omega)
$$

for all $J \geqslant 0$. By a similar argument, there is a $\tilde{b} \in S^{-m}(\Omega)$ such that $|\zeta|^{-J}(p \# \tilde{b}-1)$ $\in S^{-2 J}(\Omega)$. Hence $|\zeta|^{-J}(p \# b-1) \in S^{-2 J}(\Omega)$, for all $J \geqslant 0$.

LEMMA 4. If $p$ satisfies the hypotheses of Lemma 1, then there is $a b \in S^{-m}\left(\mathcal{G}^{*}\right)$ such that $\langle\zeta\rangle^{-J}(b \# p-1)$ and $\langle\zeta\rangle^{-J}(p \# b-1)$ are in $S^{-2 J}\left(\mathcal{G}^{*}\right)$ for all $J$. If, in addition, $p \in \tilde{S}^{m}\left(\mathcal{G}^{*}\right)$, then $b \in \tilde{S}^{-m}\left(\mathcal{G}^{*}\right)$, and for all $\alpha, \beta$ and $J,\langle\zeta\rangle^{-J} D_{\eta}^{\alpha} D_{\zeta}^{\beta}(b \# p-1)$ and $\langle\zeta\rangle^{-J} D_{\eta}^{\alpha} D \xi(p \# b-1)$ are in $S^{-2 J-|\alpha|-\varepsilon \beta|\beta|}\left(\mathcal{G}^{*}\right)$.

Proof. The proof is the same as for Lemma 3, replacing $|\zeta|$ by $\langle\zeta\rangle$ and $\Omega$ by $\mathcal{G}^{*}$ throughout.

We next give a parametrization of the infinite-dimensional irreducible unitary representations of $G$. Since $\mathcal{G}$ is of type $H$ there is a $d$ such that the bilinear form $B_{\zeta}$ has rank $2 d$ for every $\zeta \neq 0, \zeta \in \mathcal{G}_{2}^{*}$. As in [16], given $\zeta \in \mathcal{G}_{2}^{*}-\{0\}$, there is an orthonormal basis $\mathscr{B}(\zeta)=\left\{Y_{1}(\zeta), \ldots, Y_{2 d}(\zeta), \ldots, Y_{N}(\zeta)\right\}$ for $\mathcal{G}_{1}$ such that

$$
\begin{aligned}
\left\langle\zeta,\left[Y_{j+d}(\zeta), Y_{j}(\zeta)\right]\right\rangle & =\lambda_{j}(\zeta)>0 \text { for } j \leqslant d, \text { and } \\
\left\langle\zeta,\left[Y_{j}(\zeta), Y_{k}(\zeta)\right]\right\rangle & =0 \quad \text { for all other } j \text { and } k, k \leqslant j .
\end{aligned}
$$

Given $\zeta_{0} \in \mathcal{G}_{2}^{*}-\{0\}$ we may assume that the elements of the ordered basis $\mathscr{B}(\zeta)$ are chosen smoothly on some open neighborhood of $\zeta_{0}$. Also, if $r>0$, then we may assume that

$$
Y_{j}(r \zeta)=Y_{j}(\zeta) \quad \text { and } \quad \lambda_{j}(r \zeta)=r \lambda_{j}(\zeta)
$$


Thus, $\mathcal{G}_{2}^{*}-\{0\}$ can be covered by finitely many open cones $\Gamma^{(1)}, \ldots, \Gamma^{(\nu)}$ such that there are smooth functions $\lambda_{j}^{(k)}: \Gamma^{(k)} \rightarrow \mathbf{R}$ and $Y_{j}^{(k)}: \Gamma^{(k)} \rightarrow \mathcal{G}_{1}$ for which $\Re^{(k)}(\zeta)=$ $\left\{Y_{1}^{(k)}(\zeta), \ldots, Y_{N}^{(k)}(\zeta)\right\}$ satisfies $(2.13)$ and $(2.14)$ for all $\zeta \in \Gamma^{(k)}$ and such that

$$
\lambda_{j}^{(k)}(\zeta) \geqslant c|\zeta| \quad \text { for } 1 \leqslant j \leqslant d, \zeta \in \Gamma^{(k)}
$$

where $c>0$ is constant, for $1 \leqslant k \leqslant \nu$.

Let $\Gamma$ be any one of the cones just described. As shown in [16], given $\zeta \in \Gamma$ and $\rho \in \mathbf{R}^{N-2 d}$ there is an irreducible unitary representation, which we denote $\tilde{\pi}_{\rho \zeta}$, of $G$ on $L^{2}\left(\mathbf{R}^{d}\right)$ such that

$$
\begin{aligned}
\tilde{\pi}_{\rho \zeta}\left(Y_{j}(\zeta)\right) f(t) & =\lambda_{j}(\zeta) t_{j} f(t), \quad 1 \leqslant j \leqslant d, \\
\tilde{\pi}_{\rho \zeta}\left(Y_{j+d}(\zeta)\right) f(t) & =-i \partial f / \partial t_{j}, \quad 1 \leqslant j \leqslant d, \\
\tilde{\pi}_{\rho \zeta}\left(Y_{j+2 d}(\zeta)\right) f(t) & =|\zeta|^{1 / 2} \rho_{j} f(t), \quad 0<j \leqslant N-2 d .
\end{aligned}
$$

Let $U_{\zeta} f(t)=f\left(\lambda_{1}(\zeta)^{1 / 2} t_{1}, \ldots, \lambda_{d}(\zeta)^{1 / 2} t_{d}\right) \Pi_{j=1}^{d} \lambda_{j}(\zeta)^{1 / 4}$ and let $\pi_{\rho \zeta}=U_{\zeta}^{-1} \tilde{\pi}_{\rho \zeta} U_{\zeta}$. Then

$$
\begin{gathered}
\pi_{\rho \zeta}\left(Y_{j}(\zeta)\right)=\lambda_{j}(\zeta)^{1 / 2} t_{j} f(t), \quad 1 \leqslant j \leqslant d, \\
\pi_{\rho \zeta}\left(Y_{j+d}(\zeta)\right)=-i \lambda_{j}(\zeta)^{1 / 2} \partial f / \partial t_{j}, \quad 1 \leqslant j \leqslant d, \\
\pi_{\rho \zeta}\left(Y_{j+2 d}(\zeta)\right)=|\zeta|^{1 / 2} \rho_{j} f(t), \quad 0<j \leqslant N-2 d .
\end{gathered}
$$

Define the smooth bijection $\xi: \mathbf{R}^{2 d} \times \mathbf{R}^{N-2 d} \times \Gamma \rightarrow \mathcal{G}_{1}^{*} \times \Gamma$ by

$$
\xi(w, \rho, \zeta)=\left(\sum_{j=1}^{2 d} w_{j} Y_{j}(\zeta)^{*}+\sum_{j=1}^{N-2 d} \rho_{j} Y_{j+2 d}(\zeta)^{*}, \zeta\right)
$$

where $\left\{Y_{1}(\zeta)^{*}, \ldots, Y_{N}(\zeta)^{*}\right\}$ is the basis dual to $\mathscr{B}(\zeta)$. Given $p \in S^{m}\left(\mathcal{G}_{1}^{*} \times \Gamma\right)$ define $p^{\prime}$ on $\mathbf{R}^{N} \times \Gamma$ by

$$
p^{\prime}(w, \rho, \zeta)=p(\xi(w, \rho, \zeta))
$$

Given $(w, \rho, \zeta) \in \mathbf{R}^{N} \times \Gamma$ the unit vectors $\left\{Y_{1}(\zeta)^{*}, \ldots, Y_{2 d}(\zeta)^{*}\right\}$ form a basis for the subspace parallel to the orbit containing $\xi(w, \rho, \zeta)$. Hence, there exist $x_{j}=x_{j}(\zeta)$ such that $Y_{j}(\zeta)^{*}=\left(\operatorname{ad} x_{j}\right)^{*} \xi(w, \rho, \zeta)$. Since $D_{w_{j}} p^{\prime}(w, \rho, \zeta)=D_{x_{j}} p(\xi(w, \rho, \zeta))$, it follows that $p \in S^{m}\left(\mathcal{G}_{1}^{*} \times \Gamma\right)$ if and only if for all $\alpha \in \mathbf{N}^{2 d}, D_{w}^{\alpha} p^{\prime}$ is continuous on $\mathcal{G}_{1}^{*} \times \Gamma$ and

$$
\left|D_{w}^{\alpha} p^{\prime}(w, \rho, \zeta)\right| \leqslant C_{\alpha}\left(|w|^{2}+|\rho|^{2}+|\zeta|+1\right)^{(m-|\alpha|) / 2} .
$$

If $\Omega \subset \mathcal{G}^{*}$, let $\bar{S}^{m}(\Omega)$ denote those functions $p$ for which (1.1) holds (i.e. (2.19) holds for $p^{\prime}$ ), but for which the derivatives $D_{x_{1}} \cdots D_{x_{k}} p$ are not necessarily continuous.

Let $\psi_{\rho \zeta}=\psi_{\pi}$ for $\pi=\pi_{\rho \zeta}$. Then $\psi_{\rho \zeta}: \mathbf{R}^{2 d} \rightarrow \mathcal{G}^{*}$ is given by

$$
\psi_{\rho \zeta}(w)=\xi\left(\lambda(\zeta)^{1 / 2} w,|\zeta|^{1 / 2} \rho, \zeta\right) \text {. }
$$


Let $p_{\rho \zeta}$ be the symbol of the pseudodifferential operator $\pi_{\rho \zeta}(p)$; that is, $p_{\rho \zeta}(w)=$ $p \circ \psi_{\rho \zeta}(w)=p^{\prime}\left(\lambda(\zeta)^{1 / 2} w,|\zeta|^{1 / 2} \rho, \zeta\right) \cdot p^{\prime}$ satisfies (2.19) if and only if

$$
\left|D^{\alpha} p_{\rho \zeta}(w)\right| \leqslant C_{\alpha} \lambda(\zeta)^{\alpha / 2}\left[|\zeta|\left(\left|\lambda(\zeta /|\zeta|)^{1 / 2} w\right|^{2}+|\rho|^{2}+1+|\zeta|^{-1}\right)\right]^{(m--|\alpha|) / 2}
$$

for all $(w, \rho, \zeta) \in \mathbf{R}^{N} \times \Gamma$.

Since $\lambda_{j}(\zeta /|\zeta|)=|\zeta|^{-1} \lambda_{j}(\zeta)$ and $\lambda_{j}(\zeta / \mid \zeta)^{-1}$ are both bounded on $\Gamma$ by $(2.15)$ it follows that $p \in \bar{S}^{m}\left(\mathcal{G}_{1}^{*} \times \Gamma\right)$ if and only if for all $\alpha \in \mathbf{N}^{2 d}$ there exists $C_{\alpha}$ such that

$$
\left|D^{\alpha} p_{\rho \zeta}(w)\right| \leqslant C_{\alpha}|\zeta|^{m / 2}\left(|w|^{2}+|\rho|^{2}+1+|\zeta|^{-1}\right)^{(m-|\alpha|) / 2}
$$

for all $(w, \rho, \zeta) \in \mathbf{R}^{N} \times \Gamma$.

Given $c>0$, we let $\Gamma_{c}=\{\zeta \in \Gamma:|\zeta|>c\}$ and $\Gamma_{c}^{\prime}=\{\zeta \in \Gamma:|\zeta|<c\}$.

LEMMA 5. Given $c>0$, the following are equivalent:

(a) $|\zeta|^{-k} r \in \bar{S}^{-2 k}\left(\mathcal{G}_{1}^{*} \times \Gamma_{c}\right)$ for all $k \in \mathbf{N}$.

(b) For all $m \in \mathbf{N},\left\{\langle\rho\rangle^{m} \pi_{\rho \zeta}(r): \rho \in \mathbf{R}^{N-2 d}, \zeta \in \Gamma_{c}\right\}$ is an equicontinuous family of operators from $\mathcal{S}^{*}\left(\mathbf{R}^{d}\right)$ to $\mathcal{S}\left(\mathbf{R}^{d}\right)$.

Proof. By (2.20), (a) is equivalent to

$$
\left|D^{\alpha} r_{\rho \zeta}(w)\right| \leqslant C_{\alpha, k}\left(|w|^{2}+|\rho|^{2}+1\right)^{-(2 k+|\alpha|) / 2}
$$

for all $\alpha, k$ and $(w, \rho, \zeta) \in \mathbf{R}^{N} \times \Gamma_{\text {c. }}$. This, in turn, is equivalent to

$$
\left|D^{\alpha} r_{\rho \zeta}(w)\right| \leqslant C_{\alpha, j, m}\langle w\rangle^{-j}\langle\rho\rangle^{-m}
$$

for all $\alpha, j, m$ and $(w, \rho, \zeta) \in \mathbf{R}^{N} \times \Gamma_{c}$, which is equivalent to $\left\{\langle\rho\rangle^{m} r_{\rho \zeta}: \rho \in \mathbf{R}^{N-2 d}\right.$, $\left.\zeta \in \Gamma_{c}\right\}$ being bounded in $\mathcal{S}\left(\mathbf{R}^{2 d}\right)$ for all $m$. The lemma now follows from the Schwartz kernel theorem and the Banach-Steinhaus theorem.

LEMMA 6. Given $c>0$, the following are equivalent:

(a) $|\zeta|^{-k} r \in \bar{S}^{-2 k}\left(\mathcal{G}_{1}^{*} \times \Gamma_{c}^{\prime}\right)$ for all $k \in \mathbf{N}$.

(b) For all $m$ and $k \in \mathbf{N},\left\{\langle\rho\rangle^{m}|\zeta|^{-k} \pi_{\rho \zeta}(r): \rho \in \mathbf{R}^{N-2 d}, \zeta \in \Gamma_{c}^{\prime}\right\}$ is an equicontinuous family of operators from $\varsigma^{*}\left(\mathbf{R}^{d}\right)$ to $\varsigma_{\left(\mathbf{R}^{d}\right)}$.

Proof. By (2.20), (a) is equivalent to

$$
\left|D^{\alpha} r_{\rho \zeta}(w)\right| \leqslant C_{\alpha, k}\left(|w|^{2}+|\rho|^{2}+1+|\zeta|^{-1}\right)^{-(2 k+|\alpha|) / 2}
$$

for all $\alpha, k$ and $(w, \rho, \zeta) \in \mathbf{R}^{N} \times \Gamma_{c}^{\prime}$. Since $|\zeta|$ is bounded on $\Gamma_{c}^{\prime}$, this is equivalent to

$$
\left|D^{\alpha} r_{\rho \zeta}(w)\right| \leqslant C_{\alpha, k, m}|\zeta|^{k}\left(|w|^{2}+|\rho|^{2}+1\right)^{-m}
$$

for all $\alpha, k, m$ and all $(w, \rho, \zeta) \in \mathbf{R}^{N} \times \Gamma_{c}^{\prime}$. The remainder of the proof is the same as for Lemma 5 .

For $\rho \in \mathbf{R}^{N-2 d}$ define $\chi_{\rho}$ on $\mathbf{R}^{d} \times \mathbf{R}^{d}$ by

$$
\chi_{\rho}(t, \tau)=\left(|t|^{2}+|\tau|^{2}+|\rho|^{2}+1\right)^{1 / 2} .
$$


Then $\left(\chi_{\rho}, 1\right)$ is a pair of global coercive weight functions as defined in Beals [1]. For $k \in \mathbf{R}$ let $S_{\chi_{\rho}}^{k}\left(\mathbf{R}^{2 d}\right)$ be the set of smooth functions $p$ defined on $\mathbf{R}^{2 d}$ such that for all $\alpha \in \mathbf{N}^{d}$ and $\beta \in \mathbf{N}^{d}$

$$
\left|D_{t}^{\beta} D_{\tau}^{\alpha} p(t, \tau)\right| \leqslant C_{\alpha} \chi_{\rho}(t, \tau)^{k-|\alpha|} .
$$

Let $H_{\chi_{\rho}}^{k}\left(\mathbf{R}^{d}\right)$ be the corresponding Sobolev space, with norm denoted \|\|$_{k, \rho}$. We will simply write $\chi$ for $\chi_{0}$.

For $\zeta \in \mathcal{G}_{2}^{*}-\{0\}$ and $\rho \in \mathbf{R}^{N-2 d}$ there is a $c>0$, depending on $\rho$ and $\zeta$, such that

$$
c \chi(w) \leqslant \Phi\left(\psi_{\rho \zeta}(w)\right) \leqslant c^{-1} \chi(w) .
$$

If $p \in S_{0}^{m}\left(\mathcal{S}^{*}\right)$ satisfies the hypotheses of Lemma 1 , then by (2.4) there exist $C$ and $c>0$ depending on $\rho$ and $\zeta$ such that

$$
\left|p_{\rho \zeta}(w)\right| \geqslant c \chi(w)^{m} \quad \text { if }|w| \geqslant C .
$$

The next lemma follows from (2.22) and theorems in $§ 7$ of [1].

Lemma 7. Let $p \in S_{0}^{m}\left(\mathcal{G}^{*}\right)$ satisfy the hypotheses of Lemma 1 . Let $\pi=\pi_{\rho \zeta}$ for $\zeta \in \mathcal{G}_{2}^{*}-\{0\}$ and $\rho \in \mathbf{R}^{N-2 d}$. Then $\pi(p)$ is a Fredholm operator from $H_{\chi}^{m+k}\left(\mathbf{R}^{d}\right)$ to $H_{\chi}^{k}\left(\mathbf{R}^{d}\right)$ for all $k$, and the kernel of $\pi(p): H_{\chi}^{m+k}\left(\mathbf{R}^{d}\right) \rightarrow H_{\chi}^{k}\left(\mathbf{R}^{d}\right)$ is contained in $\delta\left(\mathbf{R}^{d}\right)$. If $\pi(p)$ is injective on $\delta\left(\mathbf{R}^{d}\right)$, then $\pi(p)$ has a left inverse which is a pseudodifferential operator with symbol in $S_{\chi}^{-m}\left(\mathbf{R}^{2 d}\right)$.

Lemma 8. Suppose $m \geqslant 0$ and $c>0$. Let $p \in S_{0}^{m}\left(\mathcal{G}^{*}\right)$ satisfy the hypotheses of Lemma 1. Suppose that both $\pi(p)$ and $\pi\left(p_{0}\right)$ are injective on $\delta_{\pi}$ for all $\pi=\pi_{\rho \zeta}$ such that $|\zeta| \geqslant c$. Then there is a $C$ such that

$$
\|u\| \leqslant C|\zeta|^{-m / 2}\left\|\pi_{\rho \zeta}(p) u\right\|
$$

for all $\rho \in \mathbf{R}^{N-2 d}, \zeta \in \mathcal{G}_{2}^{*},|\zeta| \geqslant c$, and $u \in L^{2}\left(\mathbf{R}^{d}\right)$, where \|\| denotes the $L^{2}$-norm.

Proof. Let $b \in S^{-m}\left(\mathcal{G}^{*}\right)$ have the properties stated in Lemma 4. Let $P_{\rho \zeta}=\pi_{\rho \zeta}(p)$, $B_{\rho \zeta}=\pi_{\rho \zeta}(b)$ and $R_{\rho \zeta}=I-B_{\rho \zeta} P_{\rho \zeta}$. By Lemma 5 there is a $C_{1}$ such that $|\rho|\left\|R_{\rho \zeta} u\right\|$ $\leqslant C_{1}\|u\|$ for all $|\zeta| \geqslant c$ and all $\rho$. It follows from (2.20) that $|\zeta|^{m / 2} b_{\rho \zeta}$ is bounded in $S_{\chi}^{0}\left(\mathbf{R}^{2 d}\right)$. Thus,

$$
\|u\| \leqslant|\zeta|^{-m / 2}\left\||\zeta|^{m / 2} B_{\rho \zeta} P_{\rho \zeta} u\right\|+\left\|R_{\rho \zeta} u\right\| \leqslant C|\zeta|^{-m / 2}\left\|P_{\rho \zeta} u\right\|+\|u\| / 2
$$

if $|\rho| \geqslant 2 C_{1}$. Hence, it suffices to prove (2.23) for $|\rho| \leqslant 2 C_{1}$.

Suppose (2.23) does not hold. Then there exist sequences $u_{j} \in L^{2}, \zeta_{j} \in \mathcal{G}_{2}^{*},\left|\zeta_{j}\right| \geqslant c$, and $\rho_{j} \rightarrow \rho_{\infty} \in \mathbf{R}^{N-2 d}$ such that $\left\|u_{j}\right\|=1$ and

$$
\left|\zeta_{j}\right|^{-m / 2}\left\|\pi_{\rho_{j} \zeta_{j}}(p) u_{j}\right\| \leqslant 1 / j
$$

Choose $F \in C^{\infty}(\mathbf{R})$ such that $F(r) \equiv 1$ if $|r| \geqslant 2$ and $F(r) \equiv 0$ if $|r| \leqslant 1$. Let $c^{\prime}=c_{1} c\left(1+c^{2}\right)^{-1 / 2}$ where $c_{1}$ is the $c$ of Lemma 1 . If $\left\{\zeta_{j}\right\}$ is bounded let $\phi(\eta, \zeta)=$ $F\left(c^{\prime} \Phi(\eta, \zeta)^{2}|\zeta|^{-1}\right)$, while if $\left\{\zeta_{j}\right\}$ is unbounded let $\phi(\eta, \zeta)=F\left(c^{\prime}\|\eta, \zeta\|^{2}|\zeta|^{-1}\right)$. By (2.2) there is a $C_{1}>c^{\prime}$ such that $p(\eta, \zeta) \geqslant c \Phi(\eta, \zeta)^{m}$ if $c^{\prime}\|\eta, \zeta\|^{2}|\zeta|^{-1} \geqslant 1$ and $|\zeta| \geqslant C_{1}$. If $\left\{\zeta_{j}\right\}$ is unbounded, by passing to a subsequence we may assume that 
$\left|\zeta_{j}\right| \geqslant C_{1}$ for all $j$. Let $\Gamma=\left\{\zeta \in \mathcal{G}_{2}^{*}:|\zeta| \geqslant C_{1}\right\}$ in the unbounded case and let $\Gamma=\left\{\zeta \in \mathcal{G}_{2}^{*}:|\zeta| \geqslant c\right\}$ in the bounded case. By (2.2) and (2.4) in either the bounded or unbounded case $a=\phi p^{-1} \in S^{-m}\left(\mathcal{S}_{1}^{*} \times \Gamma\right)$.

In either case let $r=1-a \# p$. Then $|\zeta|^{-1} r \in S^{-2}\left(\mathcal{G}_{1}^{*} \times \Gamma\right)$. If $\left\{\zeta_{j}\right\}$ is bounded, by passing to a subsequence we may assume that there is a $\zeta_{\infty}$ such that $\zeta_{j} \rightarrow \zeta_{\infty}$, in which case we let

$$
\begin{array}{ll}
\pi_{j}=\pi_{\rho_{j} \zeta,}, & p_{j}=\left|\zeta_{j}\right|^{-m / 2} p \circ \psi_{\pi_{j}}, \\
a_{j}=\left|\zeta_{j}\right|^{m / 2} a \circ \psi_{\pi_{j}}, & r_{j}=1-a_{j} \# p_{j},
\end{array}
$$

for $1 \leqslant j \leqslant \infty$. If $\left\{\zeta_{j}\right\}$ is unbounded, then, by passing to subsequence we may assume there is an $\omega_{\infty}$ such that $\zeta_{j} /\left|\zeta_{j}\right| \rightarrow \omega_{\infty}$, in which case we define $\pi_{j}, p_{j}, a_{j}$ and $r_{j}$ by (2.25) for $1 \leqslant j<\infty$ and let $\pi_{\infty}=\pi_{\rho_{\infty} \omega_{\infty}}, p_{\infty}=p_{0} \circ \psi_{\pi_{\infty}}, a_{\infty}=\left(\phi p_{0}^{-1}\right) \circ \psi_{\pi_{\infty}}$, and $r_{\infty}=1-a_{\infty} \# p_{\infty}$. Let $P_{j}, A_{j}$ and $R_{j}$ be the pseudodifferential operators with symbols $p_{j}, a_{j}$ and $r_{j}$ for $1 \leqslant j \leqslant \infty$. By (2.20), in both the bounded and unbounded cases $\left\{p_{j}\right\}$ converges weakly to $p_{\infty}$ in $S_{\chi}^{m}\left(\mathbf{R}^{2 d}\right)$ in the sense that $\left\{p_{j}\right\}$ is bounded in $S_{\chi}^{m}\left(\mathbf{R}^{2 d}\right)$ and converges to $p_{\infty}$ in the topology of $\mathcal{E}\left(\mathbf{R}^{2 d}\right)$. Also, $\left\{a_{j}\right\}$ converges weakly to $a_{\infty}$ in $S_{\chi}^{-m}\left(\mathbf{R}^{2 d}\right)$, hence $\left\{r_{j}\right\}$ converges weakly to $r_{\infty}$ in $S_{\chi}^{-2}\left(\mathbf{R}^{2 d}\right)$. Thus $\left\{r_{j}\right\}$ converges to $r_{\infty}$ in the topology of $S_{\chi}^{0}\left(\mathbf{R}^{2 d}\right)$ and, consequently, $\left\|R_{j}-R_{\infty}\right\| \rightarrow 0$ for the operator norm on $L^{2}$. Now

$$
u_{j}=A_{j} P_{j} u_{j}+\left(R_{j}-R_{\infty}\right) u_{j}+R_{\infty} u_{j},
$$

where $\left\|A_{j} P_{j} u_{j}\right\| \leqslant C\left\|P_{j} u_{j}\right\| \rightarrow 0$ by $(2.24)$ and $\left\|\left(R_{j}-R_{\infty}\right) u_{j}\right\| \rightarrow 0$ by the preceding remark. Since $R_{\infty}$ is a compact operator on $L^{2}$ (Theorem 6.11 of [1]), by passing to a subsequence we may assume there is a $u_{\infty} \in L^{2}$ such that $u_{j} \rightarrow u_{\infty}$ in $L^{2}$. It follows from (2.24) that $\boldsymbol{P}_{\infty} u_{\infty}=0$. Lemma 7 implies that $\operatorname{ker} P_{\infty} \subset \mathcal{S}$, hence, by the hypotheses of the lemma, $u_{\infty}=0$. This contradicts $\left\|u_{j}\right\| \equiv 1$, which proves Lemma 8 .

\section{Inverses.}

Proof of Theorem 1. We first show that it suffices to prove the theorem for $m \geqslant 0$. Note that $\Phi^{2}$ satisfies the hypotheses of Theorem 1. Let $\Phi_{2 k}=\Phi^{2} \# \cdots \# \Phi^{2}$ ( $k$ times). If $p \in S_{0}^{m}\left(\mathcal{G}^{*}\right)$ satisfies the hypotheses of Theorem 1 , choose $k$ so that $2 k+m \geqslant 0$. Then $\Phi_{2 k} \# p$ satisfies the hypotheses of Theorem 1 . If $\lambda(q)$ is a left inverse for $\lambda\left(\Phi_{2 k} \# p\right)=\lambda\left(\Phi_{2 k}\right) \lambda(p)$, then $\lambda\left(q \# \Phi_{2 k}\right)$ is a left inverse for $\lambda(p)$.

Likewise, it suffices to prove the theorem under the assumption that $\pi(p)$ : $H_{\chi}^{m+k}\left(\mathbf{R}^{d}\right) \rightarrow H_{\chi}^{k}\left(\mathbf{R}^{d}\right)$ has a two-sided inverse with symbol in $S_{\chi}^{-m}\left(\mathbf{R}^{2 d}\right)$ for all $k$ and each infinite-dimensional representation $\pi$. For if $p \in S_{0}^{m}\left(\mathcal{G}^{*}\right)$ satisfies the hypotheses, then so does $\bar{p} \# p \in S_{0}^{2 m}\left(\mathcal{G}^{*}\right)$. By Lemma $7, \pi(\bar{p} \# p)=\pi(p)^{*} \pi(p): H_{\chi}^{-k}\left(\mathbf{R}^{d}\right) \rightarrow$ $H_{\chi}^{-k-2 m}\left(\mathbf{R}^{d}\right)$ has a left inverse $Q$ with symbol in $S_{\chi}^{-2 m}\left(\mathbf{R}^{2 d}\right)$. Hence, $Q^{*}$ is a right inverse for $\pi(p)^{*} \pi(p)$ and $Q^{*}=Q$ when both are considered as operators from $H_{\chi}^{2 m+k}\left(\mathbf{R}^{d}\right)$ to $H_{\chi}^{k}\left(\mathbf{R}^{d}\right)$. If $\lambda(q)$ is a left inverse for $\lambda(\bar{p} \# p)$, then $\lambda(q \# \bar{p})$ is a left inverse for $\lambda(p)$.

Suppose $\xi=(\eta, \zeta) \in \mathcal{G}_{1}^{*} \times \mathcal{S}_{2}^{*}$ with $\zeta=0$. Then $\mathcal{O}_{\xi}=\{\xi\}$ and $\pi_{\xi}(p)$ is multiplication by $p(\eta, 0)$, which is nonzero by hypothesis. Let $q(\eta, 0)=p(\eta, 0)^{-1}$. 
Recall that every infinite-dimensional irreducible unitary representation of $G$ is equivalent to exactly one of the representations $\pi_{\rho \zeta}$ with $\zeta \in \mathcal{G}_{2}^{*}-\{0\}$ and $\rho \in$ $\mathbf{R}^{N-2 d}$. Given an orbit $\theta$ of the coadjoint action of $G$ on $\mathcal{G}^{*}, \theta$ containing more than one point, let $\pi=\pi_{\rho \zeta}$ be the corresponding representation. Let $q_{\rho \zeta}$ be the Weyl symbol for the inverse of $\pi_{\rho \zeta}(p)$. Define $q$ on $\theta$ by $q b_{\theta}=q_{\rho \zeta} \circ \psi_{\rho \zeta}^{-1}$. We shall show that $q \in S^{-m}\left(\mathcal{G}^{*}\right)$.

Let $\Omega=\mathcal{G}_{1}^{*} \times\left(\mathcal{S}_{2}^{*}-\{0\}\right)$ and let $b \in S^{-m}(\Omega)$ have the properties stated in Lemma 3. Let $r=1-p \# b$ and $r^{\prime}=q-b$. Let $P_{\rho \zeta}, Q_{\rho \zeta}, B_{\rho \zeta}, R_{\rho \zeta}$ and $R_{\rho \zeta}^{\prime}$ be the pseudodifferential operators with Weyl symbols $p_{\rho \zeta}, q_{\rho \zeta}$, etc., where $p_{\rho \zeta}=p \circ \psi_{\rho \zeta}$, etc. Then

$$
R_{\rho \zeta}^{\prime}=Q_{\rho \zeta}\left(I-P_{\rho \zeta} B_{\rho \zeta}\right)=Q_{\rho \zeta} R_{\rho \zeta} .
$$

Let $\Gamma$ be any of the cones $\Gamma^{(1)}, \ldots, \Gamma^{(\nu)}$ as described before (2.15). Choose any $c_{1}>0$ and let $\Gamma_{c_{1}}^{\prime}=\left\{\zeta \in \Gamma:|\zeta|<c_{1}\right\}$. By Lemma 6, $\left\{|\zeta|^{-1} R_{\rho \zeta}: \rho \in \mathbf{R}^{N-2 d}, \zeta \in \Gamma_{c_{1}}^{\prime}\right\}$ is equicontinuous from $\delta^{*}$ to $\delta$, where $\delta=\delta\left(\mathbf{R}^{d}\right)$, and hence from $L^{2}$ to $L^{2}$. Thus $\left\|R_{\rho \xi}\right\| \leqslant C|\zeta|$, and consequently there is a $c, 0<c<c_{1} / 2$, such that if $\zeta \in \Gamma_{2 c}^{\prime}$, then $I-R_{\rho \zeta}: L^{2} \rightarrow L^{2}$ has an inverse and $\left\{\left(I-R_{\rho \zeta}\right)^{-1}: \rho \in \mathbf{R}^{N-2 d}, \zeta \in \Gamma_{2 c}^{\prime}\right\}$ is equicontinuous from $L^{2}$ to $L^{2}$. Since $\left(I-R_{\rho \zeta}\right)^{-1}=R_{\rho \zeta}\left(I-R_{\rho \zeta}\right)^{-1}+I$ it follows from Lemma 6 that $\left\{\left(I-R_{\rho \zeta}\right)^{-1}: \rho \in \mathbf{R}^{N-2 d}, \zeta \in \Gamma_{2 c}^{\prime}\right\}$ is equicontinuous from $\delta$ to $\delta$. Since $b \in S^{-m}$, it follows from (2.20) that $\left\{|\zeta|^{m / 2} b_{\rho \zeta}: \rho \in \mathbf{R}^{N-2 d}, \zeta \in \Gamma_{2 c}^{\prime}\right\}$ is bounded in $S_{\chi}^{-m}\left(\mathbf{R}^{2 d}\right)$. Hence, $\left\{|\zeta|^{m / 2} B_{\rho \zeta}: \rho \in \mathbf{R}^{N-2 d}, \zeta \in \Gamma_{2 c}^{\prime}\right\}$ is equicontinuous from $\delta$ to $\delta$. Note that

$$
B_{\rho \zeta}\left(I-R_{\rho \zeta}\right)^{-1}=Q_{\rho \zeta} P_{\rho \zeta} B_{\rho \zeta}\left(I-R_{\rho \zeta}\right)^{-1}=Q_{\rho \zeta} .
$$

By (3.1),

$$
\langle\rho\rangle^{j}|\zeta|^{-k+m / 2} R_{\rho \zeta}^{\prime}=|\zeta|^{m / 2} B_{\rho \zeta}\left(I-R_{\rho \zeta}\right)^{-1}\langle\rho\rangle^{j}|\zeta|^{-k} R_{\rho \zeta} .
$$

Thus, for all positive integers $j$ and $k,\left\{\langle\rho\rangle^{j}|\zeta|^{-k+m / 2} R_{\rho \zeta}^{\prime}: \rho \in \mathbf{R}^{N-2 d}, \zeta \in \Gamma_{2 c}^{\prime}\right\}$ is equicontinuous from $\delta *$ to $\delta$, and, hence, by Lemma 6

$$
|\zeta|^{-k+m / 2} r^{\prime} \in \bar{S}^{-2 k}\left(\mathcal{G}_{1}^{*} \times \Gamma_{2 c}^{\prime}\right)
$$

for all $k$.

Let $\Gamma_{c}=\{\zeta \in \Gamma:|\zeta|>c\}$. By Lemma 8, $\left\{|\zeta|^{m / 2} Q_{\rho \zeta}: \rho \in \mathbf{R}^{N-2 d}, \zeta \in \Gamma_{c}\right\}$ is equicontinuous from $L^{2}$ to $L^{2}$. Let $\tilde{R}_{\rho \zeta}=I-B_{\rho \zeta} P_{\rho \zeta}$. By Lemmas 3 and 5, for all $k,\left\{\langle\rho\rangle^{k} \tilde{R}_{\rho \zeta}: \rho \in \mathbf{R}^{N-2 d}, \zeta \in \Gamma_{c}\right\}$ is equicontinuous from $\delta^{*}$ to $\delta$. Thus $\left\{|\zeta|^{m / 2}\langle\rho\rangle^{k} Q_{\rho \zeta}^{*} \tilde{R}_{\rho \zeta}^{*}: \rho \in \mathbf{R}^{N-2 d}, \zeta \in \Gamma_{c}\right\}$ is equicontinuous from $\delta^{*}$ to $\delta^{*}$ and, hence, $\left\{|\zeta|^{m / 2}\langle\rho\rangle^{k} \tilde{R}_{\rho \zeta} Q_{\rho \zeta}: \rho \in \mathbf{R}^{N-2 d}, \zeta \in \Gamma_{c}\right\}$ is equicontinuous from $\delta$ to $\delta$, for all $k$. Since $Q_{\rho \zeta}$ is a two-sided inverse for $P_{\rho \zeta}, Q_{\rho \zeta}=\tilde{R}_{\rho \zeta} Q_{\rho \zeta}+B_{\rho \zeta}$. Thus, $\left\{|\zeta|^{m / 2} Q_{\rho \zeta}\right.$ : $\left.\rho \in \mathbf{R}^{N-2 d}, \zeta \in \Gamma_{c}\right\}$ is equicontinuous from $\delta$ to $\mathcal{S}$. Since $|\zeta|^{m / 2}\langle\rho\rangle^{k} R_{\rho \zeta}^{\prime}=$ $|\zeta|^{m / 2} Q_{\rho \zeta}\langle\rho\rangle^{k} R_{\rho \zeta}$, it follows from Lemma 5 that $|\zeta|^{-k+m / 2} r^{\prime} \in \bar{S}^{-2 k}\left(\mathcal{G}_{1}^{*} \times \Gamma_{c}\right)$, for all $k$. Therefore $r^{\prime} \in \bar{S}^{-m}(\Omega)$ and, hence, $q=r^{\prime}+b \in \bar{S}^{-m}(\Omega)$.

It remains to be shown that $D_{x_{1}} \cdots D_{x_{k}} q$ is continuous for all $x_{1}, \ldots, x_{k} \in \mathcal{G}$. On $\mathcal{G}_{1}^{*} \times \Gamma$ the continuity of the functions $D_{x_{1}} \cdots D_{x_{k}} p$ is equivalent to the continuity of 
the map $(\rho, \zeta) \mapsto p_{\rho \zeta}$ from $\mathbf{R}^{N-2 d} \times \Gamma$ to $\mathscr{E}\left(\mathbf{R}^{2 d}\right)$. If $\zeta$ and $\zeta^{\prime}$ are in $\Gamma$, then

$$
q_{\rho^{\prime} \zeta^{\prime}}-q_{\rho \zeta}=q_{\rho \zeta} \#\left(p_{\rho \zeta}-p_{\rho^{\prime} \zeta^{\prime}}\right) \# q_{\rho^{\prime} \zeta^{\prime}} \cdot
$$

Since the map $(\rho, \zeta) \mapsto p_{\rho \zeta}$ is continuous from $\mathbf{R}^{N-2 d} \times \Gamma$ to $\mathcal{E}\left(\mathbf{R}^{2 d}\right)$, it follows that the map $(\rho, \zeta) \mapsto q_{\rho \zeta}$ is likewise continuous.

Finally, we show that $D_{x_{1}} \cdots D_{x_{k}} q$ is continuous when $\zeta=0$. By (2.9), $1=q \# p=$ $q p+r_{1}$ where $\left|r_{1}(\eta, \zeta)\right| \leqslant|\zeta| \Phi(\eta, \zeta)^{-2}$ for $\zeta \neq 0$. Thus,

$$
\lim _{(\eta, \zeta) \rightarrow\left(\eta_{0}, 0\right)} q(\eta, \zeta)=p\left(\eta_{0}, 0\right)^{-1}=q\left(\eta_{0}, 0\right) .
$$

Hence $q$ is continuous. Given $x \in \mathcal{G}, 0=\left(D_{x} q\right) p+q D_{x} p+D_{x} r_{1}$, from which it follows that $\lim _{(\eta, \zeta) \rightarrow\left(\eta_{0}, 0\right)} D_{x} q(\eta, \zeta)=0=D_{x} q\left(\eta_{0}, 0\right)$. By induction,

$$
\lim _{(\eta, \zeta) \rightarrow\left(\eta_{0}, 0\right)} D_{x_{1}} \cdots D_{x_{k}} q(\eta, \zeta)=0,
$$

and, hence, $D_{x_{1}} \cdots D_{x_{k}} q$ is continuous when $\zeta=0$, for all $x_{1}, \ldots, x_{k} \in \mathcal{G}$.

\section{Parametrices and hypoellipticity.}

Proof of Theorem 2. Let $c$ be any positive number. By Lemma 7 , if $|\zeta|>c$ and $\rho \in \mathbf{R}^{N-2 d}, \pi_{\rho \zeta}\left(p_{0}\right)$ has a pseudodifferential inverse; let $a_{\rho \zeta}$ be its Weyl symbol. Define $a$ on the orbit $\theta$ corresponding to $\pi_{\rho \zeta}$ by $\left.a\right|_{e}=a_{\rho \zeta} \circ \psi_{\rho \zeta}^{-1}$. It follows from the proof of Theorem 1 that $a \in S^{-m}\left(\mathcal{G}_{1}^{*} \times\{|\zeta|>c\}\right)$. By $(2.20),\left\{|\zeta|^{m / 2} a_{\rho \zeta}:|\zeta|>c\right\}$ is bounded in $S_{\chi_{\rho}}^{-m}\left(\mathbf{R}^{2 d}\right)$, uniformly in $\rho$. Thus, there is a $C$ such that

$$
\|u\|_{m, \rho} \leqslant C|\zeta|^{-m / 2}\left\|\pi_{\rho \zeta}\left(p_{0}\right) u\right\|
$$

for all $u \in H_{\chi_{\rho}}^{m}\left(\mathbf{R}^{d}\right), \rho \in \mathbf{R}^{N-2 d}$ and $|\zeta|>c$. Now $p=p_{0}+p_{1}$ where

$$
\left\|\pi_{\rho \zeta}\left(p_{1}\right) u\right\| \leqslant C_{1}|\zeta|^{(m-\varepsilon) / 2}\|u\|_{m, \rho}
$$

It follows that there is a $C$ such that if $|\zeta| \geqslant C$, then

$$
\|u\|_{m, \rho} \leqslant C|\zeta|^{-m / 2}\left\|\pi_{\rho \zeta}(p) u\right\| .
$$

For $|\zeta| \geqslant C$, let $\tilde{q}_{\rho \zeta}$ be the symbol of the inverse of $\pi_{\rho \zeta}(p)$ and define $\left.\tilde{q}\right|_{\mathcal{C}}=\tilde{q}_{\rho \zeta} \circ \psi_{\rho \zeta}^{-1}$, where $\theta$ is the orbit corresponding to $\pi_{\rho \zeta}$. Let $b \in S^{-m}\left(\mathcal{G}^{*}\right)$ have the properties stated in Lemma 4. Choose $\phi \in C_{0}^{\infty}\left(\mathcal{G}_{2}^{*}\right)$ with $\phi \equiv 1$ for $|\zeta| \leqslant 2 C$. Define $q \in S^{-m}\left(\mathcal{G}^{*}\right)$ by $q=\phi b+(1-\phi) \tilde{q}$. Since $\phi$ is constant on each orbit, $q \# p-1=\phi(b \# p-1) \in$ $S^{-k}\left(\mathcal{G}^{*}\right)$ for all $k \in \mathbf{N}$.

Let $r=q \# p-1$. To complete the proof of Theorem 2 we must show that $\lambda(r)$ : $\mathcal{E}^{*}(G) \rightarrow \mathcal{E}(G)$. For this purpose we will give a very rudimentary treatment of the even integer Sobolev spaces for $G$. More complete treatments of Sobolev spaces on nilpotent Lie groups appear in [18 and 20]. Since $\Phi^{2}$ satisfies the hypotheses of Theorem 1, there is a $\Phi_{-2} \in S^{-2}\left(\mathcal{G}^{*}\right)$ such that $\lambda\left(\Phi_{-2}\right)=\lambda\left(\Phi^{2}\right)^{-1}$. (It seems reasonable to expect that $\pi\left(\Phi^{s}\right)$ is injective for all $s \geqslant 0$, in which case the following treatment of Sobolev spaces would be valid for all $s \in \mathbf{R}$.) For integer $k>0$ let $\Phi_{2 k}=\Phi^{2} \# \cdots \# \Phi^{2}$ and $\Phi_{-2 k}=\Phi_{-2} \# \cdots \# \Phi_{-2}$. For integer $k$, let $H^{2 k}(G)$ be the completion of $\delta(G)$ with respect to the norm $\|u\|_{2 k}=\left\|\lambda\left(\Phi_{2 k}\right) u\right\|$, where \|\| is the $L^{2}$-norm. Let $H^{k}(\mathcal{G})$ denote the standard Sobolev space on the vector space $\mathcal{G}$ : $\phi \in H^{k}(\mathcal{G})$ if and only if $\left(1+|\zeta|^{2}\right)^{k / 2} \hat{\phi}(\xi) \in L^{2}$. 
If $p \in S^{2 j}\left(\mathcal{S}^{*}\right), j$ an integer, then it follows from Theorems 3.1 and 4.1 of [17] that $\lambda\left(\Phi_{2 k-2 j}\right) \lambda(p) \lambda\left(\Phi_{-2 k}\right)$ is a bounded operator on $L^{2}(G)$. Hence, $p \in S^{2 j}\left(\mathcal{G}^{*}\right)$ implies

$$
\lambda(p): H^{2 k}(G) \rightarrow H^{2 k-2 j}(G) .
$$

In particular, $u \in H^{2 k}(G)$ if and only if $P u \in L^{2}(G)$ for every left-invariant partial differential operator which is homogeneous of degree $2 k$ when $k \geqslant 0$. It follows that if $u \in H^{2 k}(G), k>0$, then $u \circ \exp \in H_{\text {loc }}^{k}(\hat{\mathcal{G}})$. As usual, the pairing

$$
\langle u, v\rangle=\int u \bar{v} d g=\int \lambda\left(\Phi_{2 k}\right) u \overline{\lambda\left(\Phi_{-2 k}\right) v} d g
$$

establishes an isomorphism between $H^{-2 k}(G)$ and the dual of $H^{2 k}(G)$. Since $\cap H_{1 \times}^{k}(\hat{S}) \subset \mathcal{E}(\mathcal{G})$, it follows that $\lambda(r): \mathcal{E}^{*}(G) \rightarrow \mathcal{E}(G)$ for any $r \in S^{-\infty}\left(\mathcal{G}^{*}\right)=$ $\cap S^{-k}\left(\hat{i}^{*}\right)$.

Define the following class of symbols: $p \in \tilde{S}^{m}\left(\mathcal{G}^{*}\right)$ if $p \in \mathcal{E}\left(\mathcal{G}^{*}\right)$ and there is an $\varepsilon$, $0<\varepsilon \leqslant 2$, such that for all $\alpha$ and $\beta$

$$
\left|D_{\eta}^{\alpha} D_{\zeta}^{\beta} p(\eta, \zeta)\right| \leqslant C_{\alpha \beta} \Phi(\eta, \zeta)^{m-|\alpha|-\epsilon \beta \mid} .
$$

COROllary 1. If $p \in \tilde{S}_{0}^{m}\left(\hat{S}^{*}\right)$ satisfies the hypotheses of Theorem 2 , then the $q$ constructed in Theorem 2 is in $\tilde{S}^{-m}\left(\hat{S}^{*}\right)$ and $\lambda(p)$ is hypoelliptic.

Proof. Let $b, \tilde{q}$ and $C$ be as in the proof of Theorem 2. By Lemma $4, b \in \tilde{S}^{-m}\left(\mathcal{G}^{*}\right)$. Let $\Gamma$ be any of the cones $\Gamma^{(1)}, \ldots, \Gamma^{(\nu)}$ described in $\S 2$, and let $\Gamma_{C}=\{\zeta \in \Gamma$ : $|\zeta|>C\}$. For any $p \in S^{m}\left(\xi_{1}^{*} \times \Gamma_{C}\right)$ define $p^{\prime}$ by (2.18) and let $L p=p^{\prime}$. Then $L$ is a bijection of $S^{m}\left(\hat{i}_{1}^{*} \times \Gamma_{C}\right)$ onto a set of functions which will be denoted $S^{m}\left(\mathbf{R}^{N} \times \Gamma_{C}\right)$. Define \# on $S^{\prime \prime}\left(\mathbf{R}^{N} \times \Gamma_{C}\right) \times S^{k}\left(\mathbf{R}^{N} \times \Gamma_{C}\right)$ by $p_{1}^{\prime} \# p_{2}^{\prime}=\left(p_{1} \# p_{2}\right)^{\prime}$.

Denote elements of $\mathbf{R}^{N}$ by $(w, \rho)$ where $w \in \mathbf{R}^{2 d}$ and $\rho \in \mathbf{R}^{N-2 d}$. For $h \in \mathbf{R}^{N-2 d}$, let $\tilde{q}_{h}^{\prime}(w, \rho, \zeta)=\tilde{q}^{\prime}(w, \rho+h, \zeta)$. As in the second paragraph of the proof of Theorem 1, we may assume $\pi(\tilde{q})$ is a two-sided inverse for $\pi(p)$ for all irreducible unitary representations $\pi=\pi_{\rho \zeta},|\zeta|>C$. Hence,

$$
\tilde{q}_{h}^{\prime}-\tilde{q}^{\prime}=\tilde{q}^{\prime} \#\left(p^{\prime}-p_{h}^{\prime}\right) \# \tilde{q}_{h}^{\prime}
$$

and, therefore,

$$
\frac{\partial \tilde{q}^{\prime}}{\partial \rho_{j}}=-\tilde{q}^{\prime} \# \frac{\partial p^{\prime}}{\partial \rho_{j}} \# \tilde{q}^{\prime} .
$$

Thus $\partial \tilde{q}^{\prime} / \partial \rho_{j} \in S^{-m-1}\left(\mathbf{R}^{N} \times \Gamma_{C}\right)$. Repeated application of (4.2) shows that $D_{\rho}^{\beta} \tilde{q}^{\prime} \in$ $S^{-m-|\beta|}\left(\mathbf{R}^{N} \times \Gamma_{C}\right)$, which yields the desired estimates (4.1) for all derivatives of $\tilde{q}$ in directions parallel to $\mathcal{S}_{1}^{*}$.

For $\zeta$ derivatives, (4.2) is not valid, but we may proceed as follows. $\pi_{\rho \zeta}(p)$ has symbol $p_{\rho \zeta}$ where $p_{\rho \zeta}(w)=p^{\prime}\left(\lambda(\zeta)^{1 / 2} w,|\zeta|^{1 / 2} \rho, \zeta\right)$. Hence for $1 \leqslant k \leqslant \operatorname{dim} \mathcal{G}_{2}$,

$$
\frac{\partial p_{\rho \zeta}}{\partial \zeta_{k}}(w)=\frac{\partial p^{\prime}}{\partial \zeta_{k}}\left(\lambda(\zeta)^{1 / 2} w,|\zeta|^{1 / 2} \rho, \zeta\right)+p_{k}^{\prime}\left(\lambda(\zeta)^{1 / 2} w,|\zeta|^{1 / 2} \rho, \zeta\right)
$$

where

$$
2 p_{k}^{\prime}(w, \rho, \zeta)=\sum \lambda_{j}(\zeta)^{-1} w_{j} \frac{\partial \lambda_{j}}{\partial \zeta_{k}} \frac{\partial p^{\prime}}{\partial w_{j}}+\sum|\zeta|^{-2} \zeta_{k} \rho_{j} \frac{\partial p^{\prime}}{\partial \rho_{j}}
$$


Let $p_{k}=L^{-1} p_{k}^{\prime}$ and $T_{k} p=p_{k}+\partial p / \partial \zeta_{k}$. It follows from (4.3) that

$$
T_{k}(a \# b)=T_{k} a \# b+a \# T_{k} b .
$$

Since each $\lambda_{j}$ is homogeneous of degree one in $\zeta$, using (2.15) we see that if $p$ satisfies $\left|D_{\eta}^{\alpha} p(\eta, \zeta)\right| \leqslant C_{\alpha} \Phi(\eta, \zeta)^{m-|\alpha|}$ for all $\alpha \in \mathbf{R}^{N}$ and all $\zeta \in \Gamma_{C}$, then

$$
|\zeta| p_{k} \in S^{m}\left(\mathcal{G}_{1}^{*} \times \Gamma_{C}\right) \text {. }
$$

Thus, if $p \in \tilde{S}^{m}\left(\mathcal{G}_{1}^{*} \times \Gamma_{C}\right)$ then

$$
|\zeta|^{\varepsilon / 2} T_{k} p \in S^{m}\left(\mathcal{G}_{1}^{*} \times \Gamma_{C}\right) .
$$

As before we may assume that $p \# \tilde{q}=\tilde{q} \# p=1$ for $|\zeta| \geqslant C$. It follows from (4.4) that $\partial \tilde{q} / \partial \zeta_{k}=-\tilde{q}_{k}-\tilde{q} \# T_{k} p \# \tilde{q}$. Hence $\mid \zeta^{\varepsilon / 2} \partial \tilde{q} / \partial \zeta_{k} \in S^{-m}\left(\mathcal{G}_{1}^{*} \times \Gamma_{C}\right)$. It follows from (2.20) that

$$
\left\{|\zeta|^{(m+\varepsilon) / 2} \pi_{\rho \zeta}\left(T_{k} \tilde{q}\right): \rho \in \mathbf{R}^{N-2 d}, \zeta \in \Gamma_{C}\right\}
$$

is equicontinuous from $L^{2}$ to $L^{2}$ and, hence, by the same argument as in $\S 3$, is also equicontinuous from $\mathcal{S}$ to $\mathcal{S}$. Let $r^{\prime}=\tilde{q}-b=\tilde{q} \# r$, where $r=1-p \# b$. Since

$$
\begin{aligned}
|\zeta|^{(m+\varepsilon) / 2}\langle\rho\rangle^{j} \pi_{\rho \zeta}\left(T_{k} r^{\prime}\right)= & |\zeta|^{(m+\varepsilon) / 2} \pi_{\rho \zeta}\left(T_{k} \tilde{q}\right)\langle\rho\rangle^{j} \pi_{\rho \zeta}(r) \\
& +|\zeta|^{m / 2} \pi_{\rho \zeta}(\tilde{q})\langle\rho\rangle^{j}|\zeta|^{\varepsilon / 2} \pi_{\rho \zeta}\left(T_{k} r\right)
\end{aligned}
$$

for all $j$, it follows from (4.6) and Lemmas 4 and 5 that

$$
|\zeta|^{-j+(m+\varepsilon) / 2} T_{k} r^{\prime} \in \bar{S}^{-2 j}\left(\mathcal{G}_{1}^{*} \times \Gamma_{C}\right)
$$

for all $j$. From (3.2) and (4.5) it follows that

$$
|\zeta|^{-j+(m+\varepsilon) / 2}\left(r^{\prime}\right)_{k} \in \bar{S}^{-2 j}\left(\mathcal{G}_{1}^{*} \times \Gamma_{C}\right)
$$

for all $j$. Taking $j=(m+\varepsilon) / 2$, it follows that $\partial r^{\prime} / \partial \zeta_{k} \in \bar{S}^{-m-\varepsilon}\left(\mathcal{G}_{1}^{*} \times \Gamma_{C}\right)$ and, consequently, $\partial \tilde{q} / \partial \zeta_{k}=\partial b / \partial \zeta_{k}+\partial r^{\prime} / \partial \zeta_{k} \in \bar{S}^{-m-\varepsilon}\left(\mathcal{G}_{1}^{*} \times \Gamma_{C}\right)$. Proceeding by induction, we obtain the desired estimates (4.1) for all $\zeta$ derivatives of $\tilde{q}$. Thus $\tilde{q} \in$ $\tilde{S}^{-m}\left(\mathcal{G}_{1}^{*} \times \Gamma_{C}\right)$ and, hence, $q \in \tilde{S}^{-m}\left(\mathcal{G}^{*}\right)$.

Since $q \in \tilde{S}^{-m}\left(\mathcal{G}^{*}\right), y^{\alpha} z^{\beta} \hat{q}(y, z) \in C^{k}(\mathcal{G})$ for $|\alpha|+\varepsilon|\beta|>2(n+k)-m$, where $n=\operatorname{dim} \mathcal{G}$ and $y \in \mathcal{G}_{1}, z \in \mathcal{G}_{2}$. Thus $\hat{q} \in C^{\infty}(\mathcal{G}-\{0\})$ and consequently $\lambda(q)$ is a pseudolocal operator. Therefore $\lambda(p)$ is hypoelliptic.

Proof of TheOREM 3. The proof is a modification of that given by Beals in [3] for partial differential operators.

Suppose $\pi$ is an irreducible unitary representation of $G$ such that $\|\eta, \zeta\| \geqslant c$ for all $(\eta, \zeta) \in \mathcal{O}_{\pi}$, and $v$ is an element of $\mathcal{S}_{\pi}$ such that $\pi(p) v=0$. For $g \in G$, define $u(g)=(v, \pi(g) v)$, where $($,$) is the L^{2}$-inner product.

For $r>0$, define $\delta_{r}: \mathcal{G}^{*} \rightarrow \mathcal{G}^{*}$ by $\delta_{r}(\eta, \zeta)=\left(r \eta, r^{2} \zeta\right)$. The transpose of $\delta_{r}$ is an automorphism of $\mathcal{G}$, also denoted $\delta_{r}$, and the exponential map pulls this over to an automorphism $\delta_{r}$ of $G$. Let $\pi_{r}=\pi \circ \delta_{r}$. If $q \in S\left(\mathcal{S}^{*}\right)$, then

$$
\begin{aligned}
\lambda(q)\left(u \circ \delta_{r}\right)(g) & =\int \hat{q} \circ \log (h) \pi_{r}\left(g^{-1} h\right) v(t) \bar{v}(t) d h d t \\
& =\left(\pi_{r}(\hat{q} \circ \log ) v, \pi_{r}(g) v\right)=\left(\pi_{r}(q) v, \pi_{r}(g) v\right) .
\end{aligned}
$$

The last equality follows from Proposition 1.2 of [17]. 
Given any $q \in \tilde{S}^{k}\left(\mathcal{S}^{*}\right)$ there exists a sequence $\left\{q_{j}\right\}$ in $\mathcal{S}\left(\mathcal{S}^{*}\right)$ such that $\left\{q_{j}\right\}$ is bounded in $\tilde{S}^{k}\left(\mathcal{G}^{*}\right)$ and converges to $q$ in $\mathscr{E}\left(\mathcal{G}^{*}\right)$. By a simple approximation argument it follows that

$$
\lambda(q)\left(u \circ \delta_{r}\right)(g)=\left(\pi_{r}(q) v, \pi_{r}(g) v\right)
$$

for all $q \in \tilde{S}^{k}\left(\mathcal{S}^{*}\right)$ and all $r>0$. In particular, $u \in C^{\infty}(G)$.

We may assume that $\pi$ is one of the representations $\pi_{\xi, V, V}$ as defined in [8 or 17], since every irreducible unitary representation is equivalent to one of these. For $r \geqslant 1, \psi_{\pi_{r}}(t, \tau)=\delta_{r} \psi_{\pi}(t, \tau)$ and $\left\|\psi_{\pi}(t, \tau)\right\| \geqslant c$ for all $(t, \tau) \in V \times V^{*}$. Hence, $p \circ \psi_{\pi_{r}}$ $=r^{m} p \circ \psi_{\pi}$ for $r \geqslant 1$, by the homogeneity hypothesis. Therefore, for $r \geqslant 1$,

$$
\lambda(p)\left(u \circ \delta_{r}\right)(g)=r^{m}\left(\pi(p) v, \pi_{r}(g) v\right)=0 .
$$

Since $P$ is hypoelliptic, $\left\{w \in C_{b}^{0}(G): \lambda(p) w=0\right\} \subset C^{1}(G)$, where $C_{b}^{0}(G)$ is the space of bounded continuous functions on $G$ with the uniform norm and $C^{1}(G)$ is the space of differentiable functions with the topology of uniform convergence of functions and first derivatives on compact sets. The inclusion is continuous by the closed graph theorem. Since $\left\{u \circ \delta_{r}: r \geqslant 1\right\}$ is a bounded subset of $\left\{w \in C_{b}^{0}(G)\right.$ : $\lambda(p) w=0\}$, given a compact subset $K$ of $G$ and $x \in \mathcal{G}$, there is a $C$ such that

$$
\left|\lambda(x)\left(u \circ \delta_{r}\right)(g)\right| \leqslant C \quad \text { for all } g \in K .
$$

If $g \in G$ and $x \in \mathcal{G}_{k}, k=1$ or 2 , then $r^{k} \lambda(x) u(g)=\lambda(x)\left(u \circ \delta_{r}\right)\left(\delta_{r}^{-1} g\right)$. For fixed $g$, $\left\{\delta_{r}^{-1} g: r \geqslant 1\right\}$ is relatively compact; hence, it follows from (4.8) that $r^{k}|\lambda(x) u(g)| \leqslant C$ for all $r \geqslant 1$. Therefore, $\lambda(x) u(g)=0$ for all $x \in \mathcal{G}_{1} \cup \mathcal{G}_{2}$ and $g \in G$. Since $\mathcal{G}_{1} \cup \mathcal{G}_{2}$ spans $\mathcal{G}$, it follows that $u$ is constant. Thus $(v, \pi(g) v)=(v, v)$, and since $\pi(g)$ is unitary it follows that $\pi(g) v=v$ for all $g$. The irreducibility of $\pi$ implies that $v=0$.

\section{REFERENCES}

1. R. Beals, A general calculus of pseudodifferential operators, Duke Math. J. 42 (1975), 1-42.

2. Characterization of pseudodifferential operators and applications, Duke Math. J. 44 (1977), 45-57.

3. , Opérateurs invariants hypoelliptiques sur un groupe de Lie nilpotent, Seminaire GoulaouicSchwartz (1976-77), exposé 19, Centre de Mathématique, Ecole Polytechnique, Palaiseau, pp. 1-8.

4. A. Dynin, An algebra of pseudodifferential operators on the Heisenberg group; symbolic calculus, Dokl. Akad. Nauk. SSSR 227 (1976), 792-795; English transl. in Soviet Math. Dokl. 17 (1976), 508-512.

5. G. Folland, Subelliptic estimates and function spaces on nilpotent Lie groups, Ark. Mat. 13 (1975), 161-207.

6. D. Geller, Local solvability and homogeneous distributions on the Heisenberg group, Comm. Partial Differential Equations 5 (1980), 475-560.

7. B. Helffer, Hypoellipticité pour des opérateurs differentiels sur les groupes de Lie nilpotents, Publications du C.I.M.E., 1977.

8. B. Helffer and J. Nourrigat, Hypoellipticité pour des groupes nilpotents de rang de nilpotence 3, Comm. Partial Differential Equations 3 (1978), 643-743.

9. Caractérization des opérateurs hypoelliptiques homogènes invariants a gauche sur un group de Lie nilpotent gradué, Comm. Partial Differential Equations 4 (1979), 899-958.

10. L. Hörmander, Hypoelliptic second order differential equations, Acta Math. 119 (1967), 147-171.

11. , The Weyl calculus of pseudo-differential operators, Comm. Pure Appl. Math. 32 (1979), 359-443.

12. P. Lévy-Bruhl, Résolubilité locale et globale d'opérateurs invariants du seconde ordre sur des groupes de Lie nilpotents, Bull. Sci. Math (2) 104 (1980), 369-391.

13. A. Melin, Parametrix constructions for some classes of right invariant differential operators on the Heisenberg group, Comm. Partial Differential Equations 6 (1981), 1363-1405. 
K. G. MILLER

14. G. Metivier, Hypoellipticité analytique sur des groupes nilpotents de rang 2, Duke Math. J. 47 (1980), 195-220.

15. K. Miller, Hypoellipticity on the Heisenberg group, J. Funct. Anal. 31 (1979), 306-320.

16. __ Parametrices for hypoelliptic operators on step two nilpotent Lie groups, Comm. Partial Differential Equations 5 (1980), 1153-1184.

17. Invariant pseudodifferential operators on two step nilpotent Lie groups, Michigan Math. J. 29 (1982), 315-328.

18. C. Rockland, Hypoellipticity on the Heisenberg group: representation-theoretic criteria, Trans. Amer. Math. Soc. 240 (1978), 1-52.

19. L. Rothschild and D. Tartakoff, Inversion of analytic matrices and local solvability of some invariant differential operators on nilpotent Lie groups, Comm. Partial Differential Equations 6 (1981), 625-650.

20. K. Saka, Besov spaces and Sobolev spaces on a nilpotent Lie group, Tôhoku Math. J. 31 (1979), $383-437$.

Department of Mathematics, Wichita State University, Wichita, Kansas 67208 